\title{
Exploiting Elastic Energy Storage for Cyclic Manipulation: Modeling, Stability, and Observations for Dribbling
}

\author{
Sami Haddadin, Kai Krieger, and Alin Albu-Schäffer
}

\begin{abstract}
For creating robots that are capable of human like performance in terms of speed, energetic properties, and robustness, intrinsic compliance is a promising design element. In this paper we investigate the effects of elastic energy storage and release for ball dribbling in terms of cycle stability. We base the analysis on error evolution, peak power performance during hand contact, and robustness with respect to varying hand stiffness. As the ball can only be controlled during contact, an intrinsically elastic hand extends the contact time and improves the energetic characteristics of the process. As a human is able to dribble blindly, we decided to focus on the case of contact force sensing only, i.e. no vision is used in our approach.
\end{abstract}

\section{INTRODUCTION}

Actively compliant robots have found their way to market with the launch of the DLR Lightweight Robot III (LWR-III) that was recently commercialized as the KUKA Lightweight Robot. These robots make it possible to dexterously manipulate objects and to interact with dynamic and (partially) unknown environments. Along this line of research the design of systems with intrinsic compliance has drawn significant attention. The approach has been motivated by the possibility to provide compliant behavior for the entire mechanical bandwidth, which is of course much larger than the one of an active compliance loop. Furthermore, good shock resistance and the ability to dynamically store and release energy are main motivations for the design choice. The latter was recently exploited for explosive motions as e.g. in throwing [1]. Based on an optimal control formulation the use of the joint elasticity enables the robot to reach link speeds that are significantly larger than the maximum motor speed. In this paper we exploit intrinsic elasticity for cyclic object manipulation based on ball dribbling with an intrinsically compliant robot.

Related hybrid object manipulation problems have been investigated for quite some time. Robot dribbling, e.g., was first introduced in [2]. The authors used a half-cylindrical tube for mapping the system to a 2-D problem. The control is reactive and pushes the ball downwards if a contact is detected. [3] utilizes a high-speed multi-fingered hand for dribbling a ping-pong ball. This experiment was used to evaluate high-speed vision for ball tracking. [4] introduced a basketball playing industrial robot, utilizing a solid plate as hand. The control mainly relies on the ball tracking vision system and achieves stability of the cycle. In [5] the authors used an elastic element for prolonging the contact time and storing elastic energy in the system based on an optimal control trajectory.

Closely related to dribbling is the classical juggling task. [6] investigated this first. It uses a mirrored and scaled version of the ball trajectory, which means that the ball has to be tracked over the entire cycle. In [7] the first blindly juggling robot was presented. [8] used only a linear motor for

The first two authors contributed equally to the work. S. Haddadin, K Krieger, and A. Albu-Schäffer are with the Institute of Robotics and Mechatronics, DLR - German Aerospace Center, Wessling, Germany, contact: sami.haddadin@dlr.de juggling without the need of active ball tracking, as the lateral motion is stabilized by the shape of the juggling paddle. In [8] the authors compared an $\mathcal{H}_{2}$ optimal controller with the previous open-loop solution, which turned out to have similar performance characteristics.

In this paper we present the analysis of an elastic dribbling robot with one DoF. This is an interesting problem in order to further understand how intrinsic elasticity can be used to achieve high-performance and energy efficiency during dynamic and/or repetitive tasks as e.g. throwing [1], walking [9] and batting [10]. Several questions arise when intrinsic elasticity is taken into account. A particularly important one is how to select the spring stiffness for optimally achieving a given task. Our aim is to analyze this by considering the dribbling problem, as this poses high demands on the robot in terms of speed, dexterity, and robustness. A rather intuitive benefit why compliance is desired, which however has not been shown up to now, is that the robot should be able to sustain longer ball contact over a longer time period compared to stiff robots. In turn, this should yield a better opportunity to robustly control the ball. Besides, a further consideration that is still to be validated is that the energy transfer between robot and ball should be a much slower process and thus require less peak power with smaller hand stiffnes. Before treating these essential questions, we first analyze the stability of the system, give an observation method for tracking the ball based on proprioceptive force sensing only, and provide a stability analysis for this observation scheme.

The paper is organized as follows. In Section II we describe the considered hybrid system and provide a solution for a periodic cycle. In Section III the stability of the calculated periodic cycle is analyzed. Then, in Sec. IV, we give a solution for tracking the ball by measuring contact forces during the hand contact phase only and also provide a stability proof for this observer. Section V gives simulation results that are compared to human dribbling and provide insight into how varying hand stiffness affects peak power, contact forces, cycle robustness, and energy flows.

\section{MODELING}

In this section we describe the considered model for analyzing the periodic dribbling task. Furthermore, we introduce the used hand trajectory and then deduce an analytic solution for the equation of motion for the ball.

\section{A. MECHANICAL MODEL}

Figure 1 depicts a schematic illustration of the considered system. The ball is modeled as a point mass $m_{B}$ and radius $r_{B}$ that can do vertical movements denoted by the ball height $z_{B}$. The hand position is $z(t)$. In this paper we consider the hand to be elastic and having zero mass. The associated stiffness $K_{H}$ is attached to the hand. Since the ball stiffness is much larger than the hand stiffness, we may assume the ball to be a rigid object during hand contact. Please note 
that we consider physical springs and not actively controlled elastic behavior, as no available robot is able to provide the necessary control bandwidth for such high-speed motions. We use this simplified robot model as we want to study the essential elements of the vertical elastic dribbling cycles. Furthermore, as the spring is considered to be much more compliant than the robot, we may assume the robot to be a position actuator.

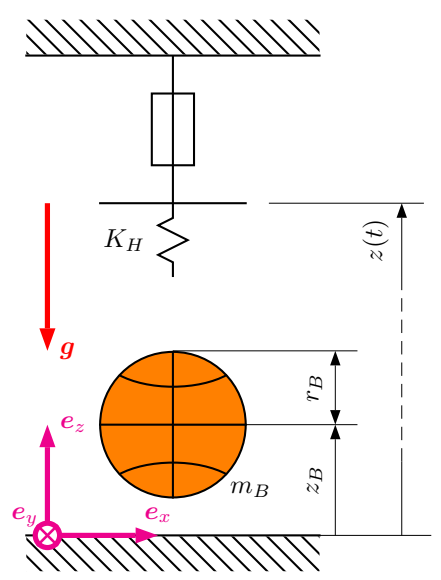

Fig. 1. Model of the considered 1-DoF dribbling task.

The ball motion is modeled as a hybrid system. One state node represents the ball being in free flight. Defining the state vector $\boldsymbol{z}_{B}:=\left[\begin{array}{ll}z_{1 B} & z_{2 B}\end{array}\right]^{T}=\left[\begin{array}{ll}z_{B} & \dot{z}_{B}\end{array}\right]^{T}$ we obtain the state space equation of motion for free flight as

$$
\dot{\boldsymbol{z}}_{B}=\boldsymbol{f}_{1}\left(\boldsymbol{z}_{B}\right)=\left[\begin{array}{c}
z_{2 B} \\
-g
\end{array}\right] \text {. }
$$

The state node during hand contact is characterized by the linear spring, which anchor point is moving with a desired position $z(t)$. Hence, during hand contact $\boldsymbol{z}_{B}$ is described by

$$
\dot{\boldsymbol{z}}_{B}=\boldsymbol{f}_{2}\left(\boldsymbol{z}_{B}, t\right)=\left[\begin{array}{c}
z_{2 B} \\
-\frac{K_{H}}{m_{B}}\left(z_{1 B}+r_{B}-z(t)\right)-g
\end{array}\right] .
$$

Based on the instantaneous ball position, the hybrid system switches between these two state nodes. Finally, if the condition $z_{1 B}+r_{B} \geq z(t)$ is fulfilled the ball is in contact with the hand.

The floor is modeled by a transition in the hybrid system, as the position before and after ground contact remains practically the same, while the velocity changes its sign and looses magnitude. The ground contact is typically in the range of $0.015 \mathrm{~s}$ (for a drop height of $1 \mathrm{~m}$ ), i.e. negligible compared to the overall dribbling cycle [11]. Hence, we introduce a coefficient of restitution (COR) that is defined as

$$
C O R:=-\frac{z_{2 B}^{+}}{z_{2 B}^{-}},
$$

with $z_{2 B}^{-}$being the velocity before and $z_{2 B}^{+}$the velocity after contact, see [12]. This instant takes place if the ball reaches the height $z_{1 B}=r_{B}$. The parameter $C O R$ is chosen to be 0.85 according to the official rules of basketball [13], where the inflation of the ball is defined based on the rebounded height.

Overall, the described model can be represented by the directed graph depicted in Fig. 2. For convenience, the ball is initialized in free flight state.

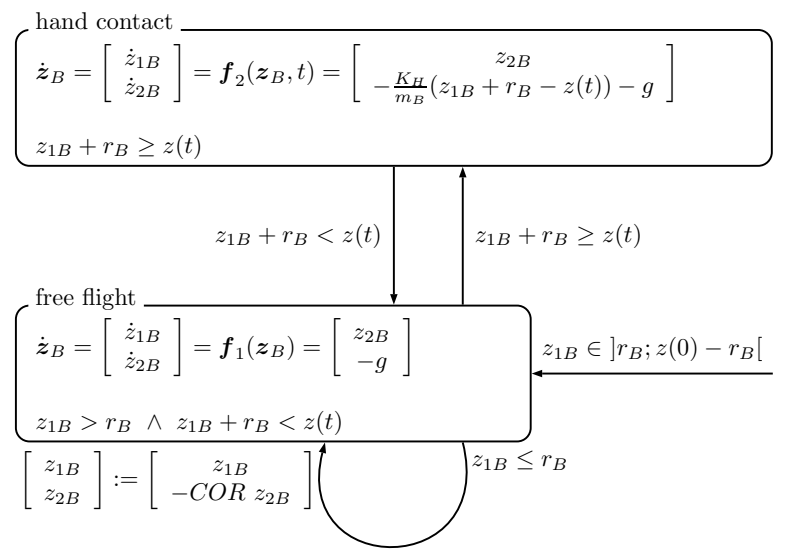

Fig. 2. Directed graph of the hybrid dribbling model.

\section{B. HAND TRAJECTORY}

In [6] the authors used a mirrored trajectory of the ball for robot juggling, which is essentially a parabola. Furthermore, negative acceleration seems desirable, as it was shown to lead to stable juggling cycles [8]. However, instead of a parabola we select a sinusoidal excitation motion of the hand, since during contact the considered system is a second order massspring complex. In addition, a positive sine half-wave has also negative acceleration but changes sign at the end so that the reversal can be carried out faster. This imposes smaller velocity and acceleration requirements on the robot. Lastly, a sine half-wave is also a good approximation for a parabola. In this paper we compose the hand trajectory from a fast and a slow sine half-wave, which frequency relation is 1 : 4. The hand trajectory is described by the three parameters amplitude $A$, offset $z_{0}$, and period time $T$, respectively the frequency $\omega=5 \pi /(4 T)$. Hence, $z(t)$ is

$$
z(t)= \begin{cases}A \sin \left(\frac{5 \pi}{4 T} t\right)+z_{0} & \text { for } t \in\left[0 ; \frac{4}{5} T\right] \\ -\frac{1}{4} A \sin \left(\frac{5 \pi}{T} t\right)+z_{0} & \text { for } t \in] \frac{4}{5} T ; T[\end{cases}
$$

which is two times continuously differentiable.

In the next subsection we derive an analytic, approximated solution for the stable cycle of the ball.

\section{ANALYTIC SOLUTION}

Figure 3 depicts the time instants at which the hybrid system changes its state node. State vectors at these time instants are denoted with a Roman number and are depicted at the top of the figure. The timely evolution of these states between the characteristic time instants are indicated in red.

At $t_{1}=0 \mathrm{~s}$ the hand contact vanishes and the initial value is $\boldsymbol{z}_{B I}:=\boldsymbol{z}_{B}(0)=\left[\begin{array}{ll}z_{1 B I} & z_{2 B I}\end{array}\right]^{T}$. The velocity is defined to be negative so that the first phase of the ball is free flight.

For the free flight phase we get

$$
\boldsymbol{z}_{F F 1}(t)=\left[\begin{array}{c}
-\frac{1}{2} g t^{2}+z_{2 B I} t+z_{1 B I} \\
-g t+z_{2 B I}
\end{array}\right] .
$$

The time instant $t_{2}^{-}$at which the ground contact occurs can be obtained by intersection of $z_{1 F F 1}(t)$ with the straight line $z=r_{B}$. We label this as the state $\boldsymbol{z}_{B I I}^{-}:=\boldsymbol{z}_{F F 1}\left(t_{2}^{-}\right)$. The floor contact is then characterized by

$$
\boldsymbol{z}_{B I I}^{+}=\left[\begin{array}{c}
z_{1 B I I}^{-} \\
-C O R z_{2 B I I}^{-}
\end{array}\right]
$$




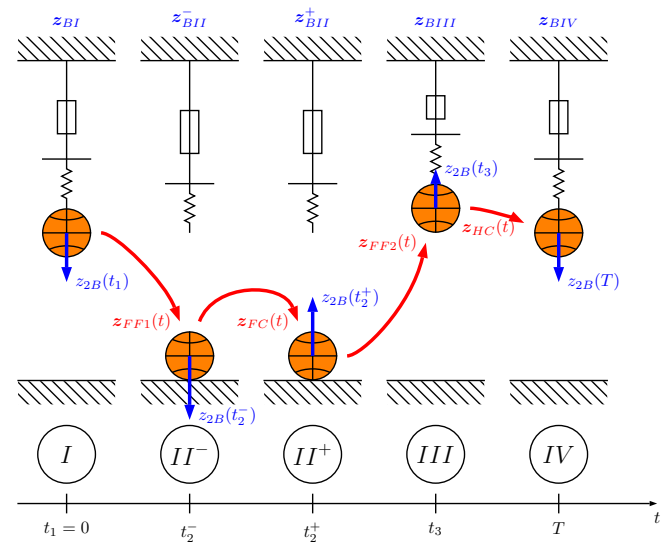

Fig. 3. State and notion conventions for the ball and hand over time are as follows. $t_{1}, \boldsymbol{z}_{B I}$ are the starting time and state. $t_{2}^{-}, \boldsymbol{z}_{B I I}^{-}$the time and state for starting floor contact, $t_{2}^{+}, \boldsymbol{z}_{B I I}^{+}$for the end of floor contact. $t_{3}, \boldsymbol{z}_{B I I I}$ are the time and state at hand contact start and $t_{4}, \boldsymbol{z}_{B I V}$ at its end.

and the solution for the second free-flight phase is

$z_{F F 2}(t)=\left[\begin{array}{c}-\frac{1}{2} g\left(t-t_{2}^{+}\right)^{2}+z_{2 B I I}^{+}\left(t-t_{2}^{+}\right)+z_{1 B I I}^{+} \\ -g\left(t-t_{2}^{+}\right)+z_{2 B I I}^{+}\end{array}\right]$.

The next time instant that has to be calculated is the start of the hand contact. As this corresponds to the intersection of a sine with a parabola no analytic solution can be provided. Therefore, we approximate the sine of the hand motion by a parabola

$$
z_{a p p r}(t)=a+b\left(t-\frac{\pi}{2 \omega}\right)^{2} \approx z(t),
$$

with the parameters $a, b$ being selected such that they minimize the error criterion

$$
J=\int_{0}^{\frac{\pi}{\omega}}\left(z_{\text {appr }}-z(t)\right) \mathrm{d} t .
$$

In addition, the cycle begins and consequently ends with the separation of the hand contact. Hence, the hand trajectory needs to be shifted along the time axis such that $z(T)=$ $z_{1 B I}+r_{B}$, which can be found as

$$
t:=t+\underbrace{\omega T-\frac{\arcsin \left(\frac{r_{B}-z_{0}+z_{1 I}}{A}\right)}{\omega}}_{t_{\text {off }}} .
$$

With (8) both, the time $t_{3}$ and the state $\boldsymbol{z}_{B I I I}:=\boldsymbol{z}_{F F 2}\left(t_{3}\right)$ are calculated. Therewith, it is possible to obtain the analytic solution for the hand contact

$$
\boldsymbol{z}_{H C}(t)=\Phi\left[\begin{array}{c}
\sin \left(\sqrt{\frac{K_{H}}{m_{B}}}\left(t-t_{3}\right)\right) \\
\cos \left(\sqrt{\frac{K_{H}}{m_{B}}}\left(t-t_{3}\right)\right) \\
\sin \left(\omega\left(t-t_{3}\right)\right) \\
\cos \left(\omega\left(t-t_{3}\right)\right)
\end{array}\right]
$$

with $\Phi$ being a $[2 \times 4]$ matrix depending on $\boldsymbol{z}_{B I I I}$ and the parameters of the hand trajectory, $m_{B}$, and $K_{H}$. With the relation $\boldsymbol{z}_{H C}(T) \stackrel{!}{=} \boldsymbol{z}_{B I}$ (where $\stackrel{!}{=}$ denotes "having to be equal to") we may inspect whether the chosen parameters result in a valid cycle. Therewith, together with the following stability analysis we are able to build regions for the set of stabilizing trajectory parameters, see Sec. V-D.
In the next section we analyze the stability of the open loop system.

\section{STABILITY ANALYSIS FOR LIMIT CYCLE}

Clearly, the system is stable for a ball lying on the floor. As we are only interested in the stability properties of dribbling limit cycles we exclude this case. For investigating stability, we use a similar method to the one described in [8]. First, we suppose that we have found parameters for a closed cycle according to Sec. II. By perturbing the initial conditions of the cycle we elaborate a mapping of the error from the cycle start to its end. For this, we use an iterative method, which idea is summarized in Fig. 4. The desired overall mapping ${ }^{I V} M_{I}$ is constructed from the concatenation of the partial mappings ${ }^{i} M_{j}$.

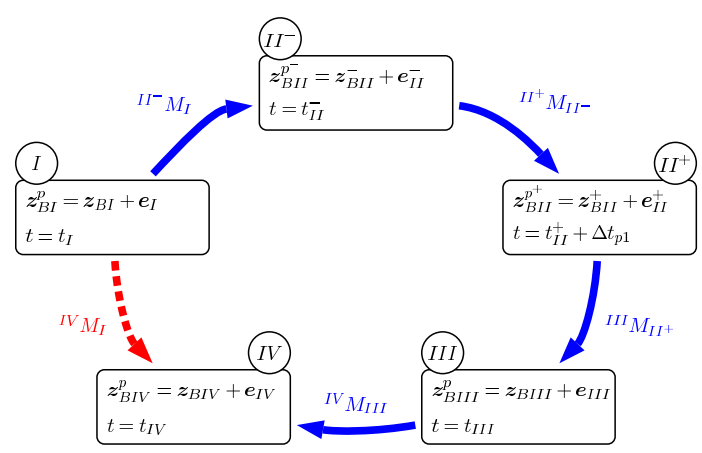

Fig. 4. Error mapping over one cycle.

\section{A. Free flight}

For free flight we define a new perturbed initial condition

$$
\boldsymbol{z}_{B I}^{p}=\boldsymbol{z}_{B I}+\boldsymbol{e}_{I}
$$

where $e_{I}=\left[\begin{array}{ll}e_{1 I} & e_{2 I}\end{array}\right]^{T}$ is the initial perturbation. Using the new initial condition (12) for the free flight we obtain a perturbed state $\boldsymbol{z}_{B I I}^{p^{-}}$at the nominal floor contact time $t_{2}^{-}$. As we assume to have small errors, we may take the linearization around the nominal unperturbed solution

$$
\begin{aligned}
\boldsymbol{z}_{B I I}^{p^{-}} & =\underbrace{\left.\boldsymbol{z}_{B I I}^{p^{-}}\right|_{\boldsymbol{e}_{I}=\mathbf{0}}}_{\boldsymbol{z}_{B I I}^{-}}+\underbrace{\left.\frac{\left.\partial \boldsymbol{z}_{B I I}^{p^{-}}\right|_{\text {III- }}}{\partial \boldsymbol{e}_{I}}\right|_{\boldsymbol{e}_{I}=\mathbf{0}}}_{\boldsymbol{e}_{I I}^{-}} \boldsymbol{e}_{I} \\
& =\boldsymbol{z}_{B I I}^{-}+\boldsymbol{e}_{I I}^{-} .
\end{aligned}
$$

The Matrix ${ }^{I I^{-}} M_{I}$ thereby maps the error $\boldsymbol{e}_{I}$ at $t_{1}$ to the error $e_{I I}^{-}$at $t_{2}^{-}$and is found to be

$$
I^{-} M_{I}=\left[\begin{array}{cc}
1 & t_{2}^{-} \\
0 & 1
\end{array}\right]
$$

\section{B. Floor contact}

Since the nominal contact time is not the real one anymore, we have to calculate the perturbed contact time. As we assume small errors, the first order approximation of the perturbed analytic solution

$$
\boldsymbol{z}_{F F 1}^{p^{l i n}}(t)=\left[\begin{array}{c}
z_{2 B I I}^{p^{-}} t+z_{1 B I I}^{p^{-}} \\
z_{2 B I I}^{p^{-}}
\end{array}\right]
$$


may be taken at the nominal contact time $t_{2}^{-}$. The time offset $\Delta t_{p 1}$ is then calculated by intersection with the floor

$$
\boldsymbol{z}_{1 F F 1}^{p^{l i n}}\left(\Delta t_{p 1}\right) \stackrel{!}{=} r_{B}
$$

The error after this small time offset $\Delta t_{p 1}$ is calculated by the difference of the first order approximation of the nominal solution after floor contact $z_{F F 2}^{l i n}(t)$ and the first order approximation of the perturbed solution $z_{F F 1}^{p^{l i n}}(t)$. This yields the error of the perturbed trajectory after the floor contact (see Fig. 5)

$$
\begin{aligned}
\boldsymbol{e}_{I I}^{+} & =\boldsymbol{z}_{F F 2}^{l i n}\left(\Delta t_{p 1}\right)-\boldsymbol{z}_{F F 1}^{p^{l i n}}\left(\Delta t_{p 1}\right) \\
& =\left[\begin{array}{c}
z_{2 B I I}^{+} \Delta t_{p 1}+z_{1 B I I}^{+} \\
z_{2 B I I}^{+}
\end{array}\right]-\left[\begin{array}{c}
z_{2 B I I}^{p^{-}} \Delta t_{p 1}+z_{1 B I I}^{p^{-}} \\
C O R z_{2 B I I}^{p^{-}}
\end{array}\right] .
\end{aligned}
$$

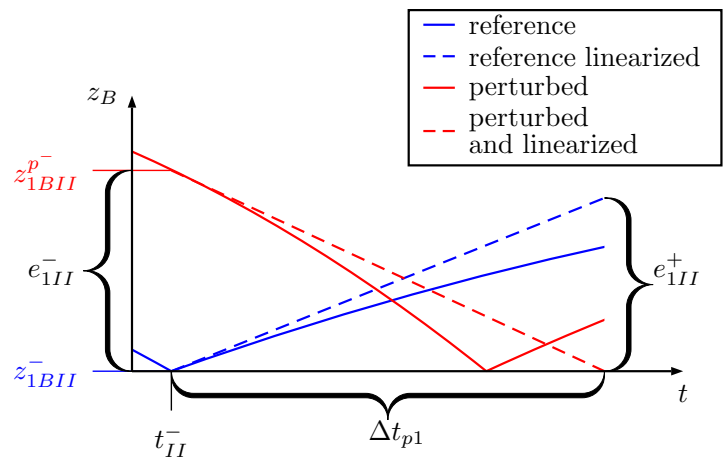

Fig. 5. Visualization of the linearized floor contact.

We obtain for this error the linearization around $e_{I I}^{-}=\mathbf{0}$

$$
e_{I I}^{+l i n}=\underbrace{\left.e_{I I}^{+}\right|_{e_{I I}^{-}=0}}_{=0}+\underbrace{\left.\frac{\partial e_{I I}^{+}}{\partial e_{I I}^{-}}\right|_{e_{I I}^{-}=\mathbf{0}}}_{=I^{I+} M_{I I^{-}}} e_{I I}^{-}
$$

with the error mapping matrix

$$
{ }^{I I^{+}} M_{I I^{-}}=\left[\begin{array}{cc}
-1 & 0 \\
0 & C O R
\end{array}\right] .
$$

\section{Free flight}

The second free flight phase is calculated from the time instant of the perturbed floor contact $t_{2}^{-}+\Delta t_{p 1}$. Therefore, we take a new initial condition for the free flight as

$$
\boldsymbol{z}_{B I I}^{p+}=\boldsymbol{z}_{F F 2}\left(t_{2}^{-}+\Delta t_{p 1}\right)+\boldsymbol{e}_{I I}^{+^{l i n}} .
$$

In the second free flight phase we get the same mapping as for the first free flight phase, see Sec. III-A. This yields

$$
\begin{aligned}
\boldsymbol{z}_{B I I I}^{p} & =\underbrace{\left.\boldsymbol{z}_{B I I I}^{p}\right|_{\boldsymbol{e}_{B I I}^{+}=\mathbf{0}}}_{\boldsymbol{z}_{B I I I}}+\underbrace{\left.\frac{\partial \boldsymbol{z}_{B I I I}^{p}}{\partial \boldsymbol{e}_{B I I}^{+}}\right|_{\boldsymbol{e}_{B I I}^{+}=\mathbf{0}}}_{=I^{I I} M_{I I^{+}}} \boldsymbol{e}_{I I}^{+l i n} \\
& =: \boldsymbol{z}_{B I I I}+\boldsymbol{e}_{I I I}
\end{aligned}
$$

The transition matrix is therefore

$$
{ }^{I I I} M_{I I^{+}}=\left[\begin{array}{cc}
1 & t_{3}-t_{2}^{-} \\
0 & 1
\end{array}\right] .
$$

With the argument from Sec. III-B a new intersection point of the hand trajectory with the ball trajectory needs to be calculated. However, this case is already included in (22), as only a negligible time increment would be added in element $\{1,2\}$ of the matrix ${ }^{I I I} M_{I I^{+}}$in (22).

\section{Hand contact}

By inserting the new initial conditions (21) at $t_{3}$ into (11) we obtain the perturbed solution for the hand contact. The linearization at $t_{4}$ yields

$$
{ }^{I V} M_{I I I}=\left[\begin{array}{cc}
\cos (c \Delta t) & \frac{1}{K_{H}} \sin (c \Delta t) \\
-K_{H} \sin (c \Delta t) & \cos (c \Delta t)
\end{array}\right]
$$

with $\Delta t=t_{4}-t_{3}$ and $c=\sqrt{K_{H} / m_{B}}$.

\section{E. Summary}

Combining (14), (19), (22) and (23) we construct the mapping of the error $\boldsymbol{e}_{I_{n}}$ of cycle $n$ to the initial error $\boldsymbol{e}_{I_{n+1}}$ of the next cycle $n+1$ by multiplication of the error mapping matrices. This yields to the difference equation

$$
\boldsymbol{e}_{I_{n+1}}=\underbrace{{ }^{I V} M_{I I I}{ }^{I I I} M_{I I^{+}}{ }^{I I^{+}} M_{I I^{-}}{ }^{I I^{-}} M_{I}}_{I V M_{I}} \boldsymbol{e}_{I_{n}} .
$$

By analyzing whether the absolute value of the eigenvalues of the matrix ${ }^{I V} M_{I}$ remains below 1 we can conclude the stability of the cycle. The eigenvalues also provide an approximation of the convergence rate of the system.

In the following section we show how the ball can be tracked by a hybrid observer that relies on force sensing only and does not require additional vision information.

\section{BALL OBSERVER}

In order to perform the dribbling task with a real robotic system it is important to be able to track the ball position. As we want to fully exploit the capabilities of proprioceptive sensing in order to support e.g. a vision system with a very robust controller it can rely on, we use a nonlinear observer that is able to reliably track the ball based on contact forces only. Then, we give a stability proof for the observer over the full cycle, for which we assume that the ball is initially in contact with the elasticity (otherwise the system is not maintaining a stable cycle and no measurement would be available).

\section{A. Observer structure}

Since we are only measuring the forces acting during the contact phase, we lack a continuous measurement. Hence, we require an observer that converges in finite time during the contact phase. A sliding mode observer is proposed in [14], that satisfies our requirement. Given a general autonoumous nonlinear system of the form

$$
\begin{aligned}
& \dot{\boldsymbol{x}}=\boldsymbol{f}(\boldsymbol{x}), \quad \boldsymbol{x} \in \mathbb{R}^{n} \\
& y=h(\boldsymbol{x}), y \in \mathbb{R} .
\end{aligned}
$$

In (25) $\mathbf{u}$ (the input) is dropped for simplicity. The observer for such a system is defined as

$$
\dot{\hat{\boldsymbol{x}}}=\left(\frac{\partial \boldsymbol{H}(\hat{\boldsymbol{x}})}{\partial \boldsymbol{x}}\right)^{-1} M(\hat{\boldsymbol{x}}) \operatorname{sgn}(\boldsymbol{V}(t)-\boldsymbol{H}(\hat{\boldsymbol{x}})),
$$


with

$$
\begin{aligned}
& \boldsymbol{H}(\boldsymbol{x})=\left[\begin{array}{lllll}
h(\boldsymbol{x}) & L_{\boldsymbol{f}} h(\boldsymbol{x}) & \ldots & L_{\boldsymbol{f}}^{n-1} h(\boldsymbol{x})
\end{array}\right]^{T} \\
& M(\hat{\boldsymbol{x}})=\operatorname{diag}\left(m_{1}(\hat{\boldsymbol{x}}) \ldots m_{n}(\hat{\boldsymbol{x}})\right) \\
& \boldsymbol{V}(t)=\left[\begin{array}{lll}
v_{1}(t) & \ldots & v_{n}(t)
\end{array}\right]^{T} .
\end{aligned}
$$

The coefficients $v_{i}$ result from the available measurement and are defined as

$$
\begin{aligned}
v_{1} & =y(t), \\
v_{i+1} & =m_{i}(\hat{\boldsymbol{x}}) \operatorname{sgn}\left(v_{i}(t)-h_{i}(\hat{\boldsymbol{x}})\right), \quad i=1(1) n-1 .
\end{aligned}
$$

[14] provides a proof that the observer converges for bounded errors in finite time depending on the gain matrix $M(\hat{\boldsymbol{x}})$. With the force acting on the robot hand, its position, and the known spring stiffness of the hand we calculate the ball position and obtain the quantity $y_{B}$ that acts as the observer input:

$$
y_{B}(t)=\frac{F}{K_{H}}-r_{B}+z(t),
$$

with $F$ being the measured force. Therewith, the observer equation during hand contact is

$$
\dot{\hat{\boldsymbol{z}}}=\boldsymbol{f}_{2}(\hat{\boldsymbol{z}}, t)+\left(\frac{\partial \boldsymbol{H}(\hat{\boldsymbol{z}})}{\partial \hat{\boldsymbol{z}}}\right)^{-1} M(\hat{\boldsymbol{z}}) \operatorname{sgn}(\boldsymbol{V}(t)-\boldsymbol{H}(\hat{\boldsymbol{z}})) \text {. }
$$

For the ball being in the free flight phase, we use a prediction step based on the model equations shown in Fig. 2. The overall hybrid observer structure, represented as a directed graph is depicted in Fig. 6.

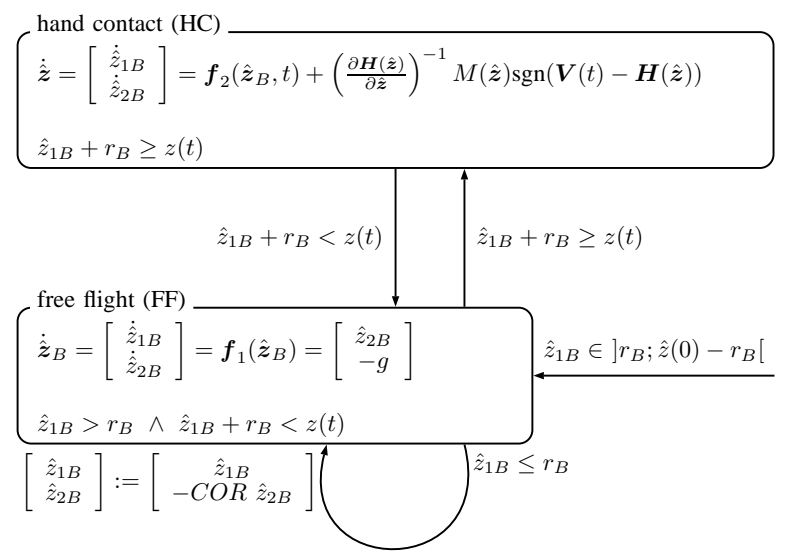

Fig. 6. Directed graph of the ball observer.

In the next subsection we give a stability analysis of the hybrid observer for the entire dribbling cycle based on the Lyapunov stability definition (up to now only the convergence during contact phase is available).

\section{B. Stability proof for the observer}

As the sliding mode observer provides convergence for bounded errors in finite time depending on the chosen gain matrix, there is no general need for a stability proof. However, as the observer converges with a constant slope, it needs to be proven that during the non-observed part the observer remains within a bounded region.

For this we consider the typical $\varepsilon, \delta$ definition of Lyapunov stability that can be found in every standard nonlinear control textbook (e.g. [15]).

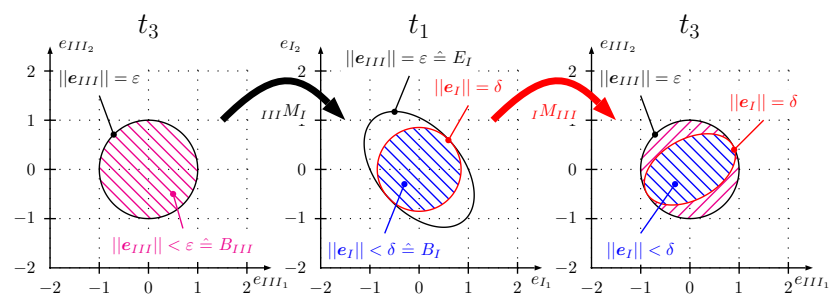

Fig. 7. $\varepsilon$ and $\delta$ regions for the proposed observer.

Definition 1 (Lyapunov stability cf. [15])

The equilibrium point $\boldsymbol{x}=0$ of (25) is

- stable if, for each $\varepsilon>0$, there is $\delta=\delta(\varepsilon)>0$ such that

$$
\|\boldsymbol{x}(0)\|<\delta \Rightarrow\|\boldsymbol{x}(t)\|<\varepsilon, \forall t \geq 0 .
$$

In the following we analyze only the time before hand contact, as the sliding mode observer itself is stable. The idea is to show the boundedness of the error mapping during non-contact phase. For this we construct the $\varepsilon, \delta$ bounds that directly represent definition 1 .

As we treat a linear system only, we may refer to the analysis from Sec. III-A to Sec. III-C for the evaluation of observer error dynamics. First, we obtain a mapping from the initial error (ball leaves hand contact) to the error at the start of the next hand contact by calculating

$$
e_{I I I}=\underbrace{{ }^{I I I} M_{I I^{+}}{ }^{I I^{+}} M_{I I^{-}}{ }^{I I^{-}} M_{I}}_{I I I M_{I}} e_{I} .
$$

This results in

$$
{ }^{I I I} M_{I}=\left[\begin{array}{cc}
-1 & -C O R t_{3} \\
0 & -C O R
\end{array}\right] .
$$

Our interest in (33) is the mapping of balls at $t_{3}$ (beginning of next hand contact) defined as

$$
B_{I I I}=\left\{\boldsymbol{x} \in \mathbb{R}^{2} \mid\|\boldsymbol{x}\|_{2}<\varepsilon\right\}
$$

back to $t_{1}$ (ball leaves previous hand contact). Let us analyze the mapping of the border of $B_{I I I}$ to $t_{1}$ by building the scalar product of $e_{I I I}$ with itself, defining that $B_{I I I}$ is the border of $e_{I I I}$. This yields to

$$
\begin{aligned}
& \boldsymbol{e}_{I I I}^{T} \boldsymbol{e}_{I I I}=\boldsymbol{e}_{I}^{T}{ }^{I I I} M_{I}^{T}{ }^{I I I} M_{I} \boldsymbol{e}_{I}=\varepsilon^{2} \\
& \Leftrightarrow \boldsymbol{e}_{I}^{T}\left[\begin{array}{cc}
1 & C O R t_{3} \\
C O R t_{3} & C O R^{2}\left(1+t_{3}^{2}\right)
\end{array}\right] \boldsymbol{e}_{I}=\varepsilon^{2} .
\end{aligned}
$$

Equation (35) corresponds to a quadric that can be transformed by a main axis transformation to an ellipse $E_{I}$ of the form

$$
\boldsymbol{e}_{I}^{T}\left[\begin{array}{cc}
\frac{1}{\varepsilon^{2} a_{1}^{2}\left(C O R, t_{3}\right)} & 0 \\
0 & \frac{1}{\varepsilon^{2} a_{2}^{2}\left(C O R, t_{3}\right)}
\end{array}\right] \boldsymbol{e}_{I}=1,
$$

where $\varepsilon a_{1}$ and $\varepsilon a_{2}$ are the lengths of the semi axes of the ellipse. Without loss of generality let $\varepsilon a_{1}$ denote the smaller semi axis. Therewith, we can define a ball at $t_{1}$ with

$$
B_{I}=\left\{\boldsymbol{x} \in \mathbb{R}^{2} \mid\|\boldsymbol{x}\|_{2}<\varepsilon a_{1}\right\},
$$

which is a region at $t_{1}$. By mapping this circle back to $t_{I I I}$ via ${ }^{I} M_{I I I}$, we construct an ellipse that lies inside $B_{I I I}$. This curve has two contact points at the major semi axis. By 
taking the open set from $B_{I I I}$ as $\varepsilon$ and the open set from $B_{I}$ as $\delta$ we get the function

$$
\delta(\varepsilon)=\varepsilon a_{1} .
$$

This approach is valid, as the region of the open set for $B_{I}$ is a subset of the obtained ellipse from the first mapping of $B_{I I I}$. Furthermore, the open set resulting from the mapping from $B_{I}$ forward is also a subset of $B_{I I I}$. Therefore, the system is stable.

Fig. 7 illustrates this approach. The left image shows the region of the circle $B_{I I I}$. For the given example we choose $\varepsilon=1$. By mapping this region forward via ${ }^{I I I} M_{I}$ we obtain the black ellipse $E_{I}$ (middle plot). The blue and red circle $B_{I}$ is the inscribed circle of the ellipse and represents $\delta$. By mapping this via ${ }^{I} M_{I I I}$, we obtain the red and blue ellipse (right plot). Finally, the hatched, blue region lies completely in the black circle, which represents $\varepsilon$.

In the following section we show human dribbling measurements, simulations for our dribbling model including the observer behavior (results from Sec. II-IV), and interesting considerations concerning the effect of varying the hand stiffness. The application of the presented concept to a full robotic system is presented in [16]. There, we also use the observer to reactively adapt the robot motion during consecutive dribbling cycles.

\section{SIMULATIONS, ENERGY CONSIDERATIONS, AND HAND STIFFNESS ANALYSIS}

In this section we show simulation results for the proposed model and observer, as well as provide a hand stiffness analysis. Furthermore, we compare our results with some dribbling measurements of a semi-pro human basketball player.

\section{A. Simulation}
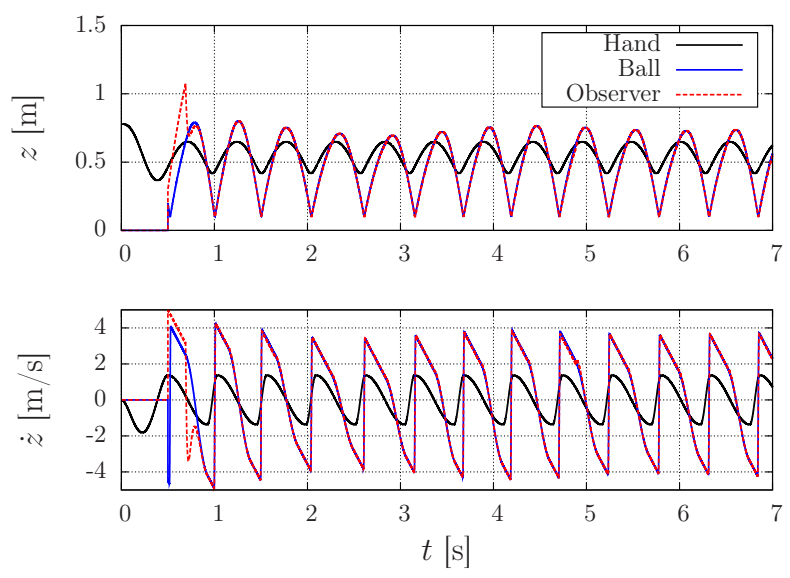

Fig. 8. Position and velocity for a sample dribbling simulation.

A sample simulation is shown in Fig. 8. The black plot depicts the hand trajectory $z$, which is shifted by an offset $-r_{B}$. For $t \leq 0.5 \mathrm{~s}$ we use a $5^{\text {th }}$ order polynomial to reach the stimulating trajectory (4). The blue curve depicts the ball motion $z_{B}$. It starts for $t>0.5 \mathrm{~s}$. From the apex heights it becomes clear that the ball stabilizes its height after some cycles. The dashed red plot denotes the ball observer position $\hat{z}_{B}$. Its initial position is set to the ball position, while its velocity has a significantly larger value than the true ball velocity. Despite this large initial discrepancy, it can be seen that the observer converges during the first contact phase.

\section{B. Human dribbling}
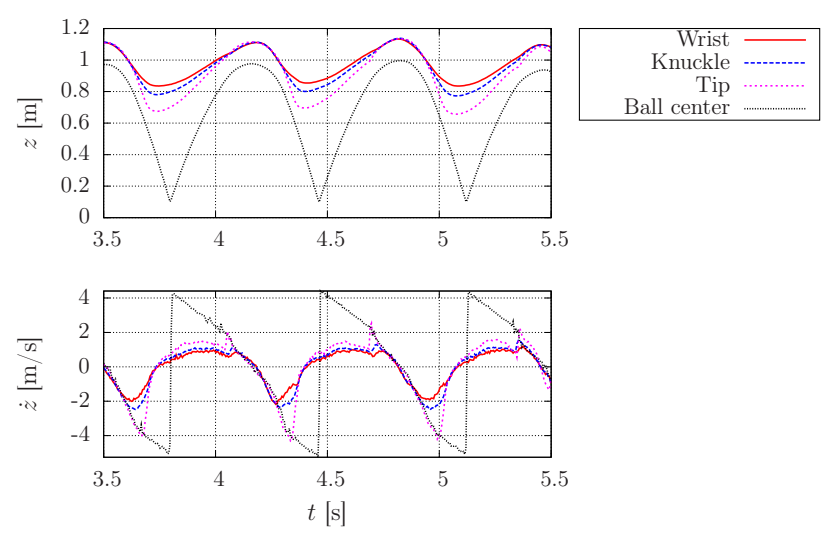

Fig. 9. Position and velocity for a sample dribbling measurement of a semi-pro human player.

Figure 9 depicts a measurement of human dribbling. The position and velocity were obtained with Vicon, a passive marker tracking system. The system consists of eight cameras running at $180 \mathrm{~Hz}$ and several markers on the human arm and ball ensured the observability over the full cycle [16]. Overall, it can be seen that the curves from Sec. V-A and the human measurement look qualitatively the same ${ }^{1}$.

\section{Energy and power considerations for varying hand stiff-} ness

Apart from achieving a stable dribbling motion it is an interesting question, which hand stiffness should be used in order to minimize peak power or increase robustness of the cycle. The last aspect is especially important for real-robots, which are generally deviating from the desired motion (in particular for such highly dynamic motions). This is particularly true for impedance controlled robots [16].

In order to analyze the effect of different hand stiffnesses, we take a closer look at the system with the trajectory used in Fig. 8 and varying hand stiffnesses. In Fig. 10(a) we find the ball position for varying stiffnesses over one cycle beginning and ending at the floor contact (after the system has already reached a stable cycle over time). The used hand trajectory is defined by $A=0.17 \mathrm{~m}, z_{0}=0.6 \mathrm{~m}$, and $T=0.52 \mathrm{~s}^{2}$ Obviously, increasing hand stiffness leads to a decreasing apex height and therewith, also to a decrease in energy level, see Fig. 10(b). As expected, the contact time increases with decreasing stiffness. This is a significant benefit one obtains from the elastic properties: more time to control the robot during hand contact. The experimental performance of this approach can be found in [16].

Interesting to notice is the energetic system behavior in terms of energy flow. We therefore consider a system, which border corresponds to the spring bearing. Hence, we obtain two energy tanks in the system. One energy portion $E_{B}$ is stored in the ball, consisting of its potential and kinetic energy. The second one is stored in the spring as potential energy $E_{S}$, see Fig. 11 (the energy flow is also depicted). Generally, there are two energy flows: 1.) $\dot{E}_{B S}$ between the two storage devices ball and spring, and 2.) $W_{S}$, which is

\footnotetext{
${ }^{1}$ Please note that the offset is not subtracted, as for human measurement it is not exactly known.

${ }^{2}$ Please note that in Fig. 10(a) the hand trajectory is displaced by $-r_{B}=$ $-0.121 \mathrm{~m}$.
} 

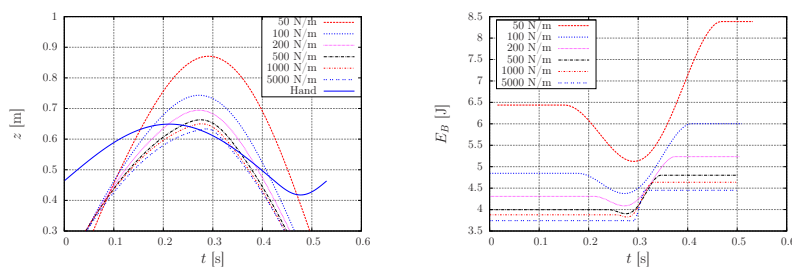

(a) Dribbling cycle for the same (b) Energy for one cycle and differhand trajectory with different hand ent hand stiffnesses. stiffnesses.

Fig. 10. Ball trajectory and energy for different hand stiffnesses at constant excitation motion (a).

the work coming from outside the system (i.e. the robot) and acts on the spring.

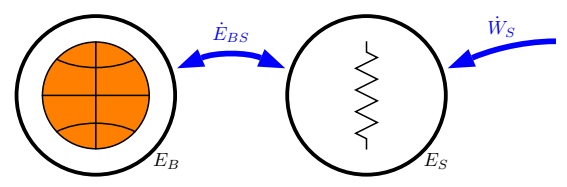

Fig. 11. Energy flow of a system consisting of a ball and a spring.

Hence, we can write the overall system energy balance as

$$
E_{B}(t)+E_{S}(t)-E_{0}=\int_{t_{0}}^{t} \dot{W}_{S}(t) \mathrm{d} t=W_{S}(t)
$$

for calculating the work to be put into the system. $E_{0}$ denotes the initial system energy.
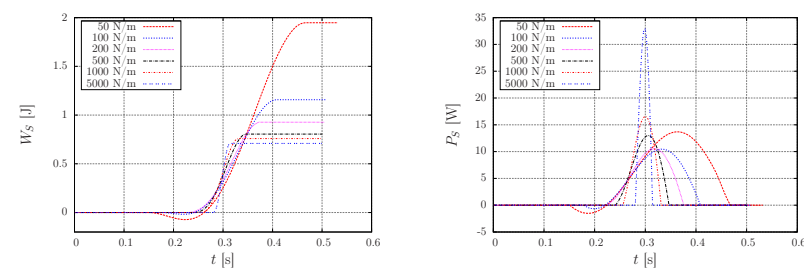

(a) Work for one cycle and different (b) Power for one cycle and different stiffnesses. stiffnesses.

Fig. 12. Work $W_{S}$ (Figure 12(a)) power $P_{s}$ (Figure 12(b)) for the dribbling cycle with varying hand stiffnesses.

The work $W_{S}$ is shown in Fig. 12(a). An interesting aspect is that for small stiffnesses the energy flows first out of the system before raising. This is due to the initial upwards hand motion at the start of the hand contact (see Fig 10(a)), which extracts potential energy from the system. Knowing the work of the system we can obtain the power

$$
P_{s}=\dot{W}_{s}
$$

that flows into the system. The power flow for the different stiffnesses is depicted in Fig. 12(b). It is obvious by looking at the apexes of the curves that a stiffness with minimal power consumption exists. This property is depicted in Fig. 13. Looking at the relation between power apex and hand spring stiffness, it is clear that an optimal hand stiffness can be selected that leads to a minimum power consumption for a given trajectory.

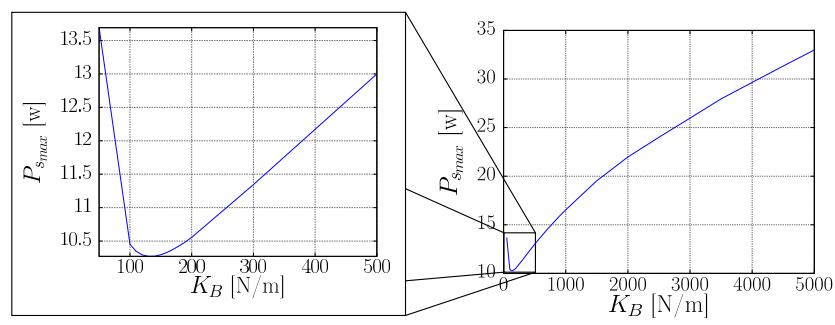

Fig. 13. Power as a function of hand stiffness $K_{H}$

\section{Stability considerations for varying hand stiffness}

Apart from the preceding energy and power analysis, we investigate which hand trajectories lead to a stable periodic cycle and their relation to hand stiffness. Figure 14 depicts the simulation analysis results for varying $A, T$, and $K_{H}$ at a constant height $z_{0}$. The stability check for obtaining the plotted regions was done by applying the equations for the periodic cycle of Sec. II-C and the according stability analysis from Sec. III. For higher hand stiffnesses we generally need smaller amplitudes and period times.

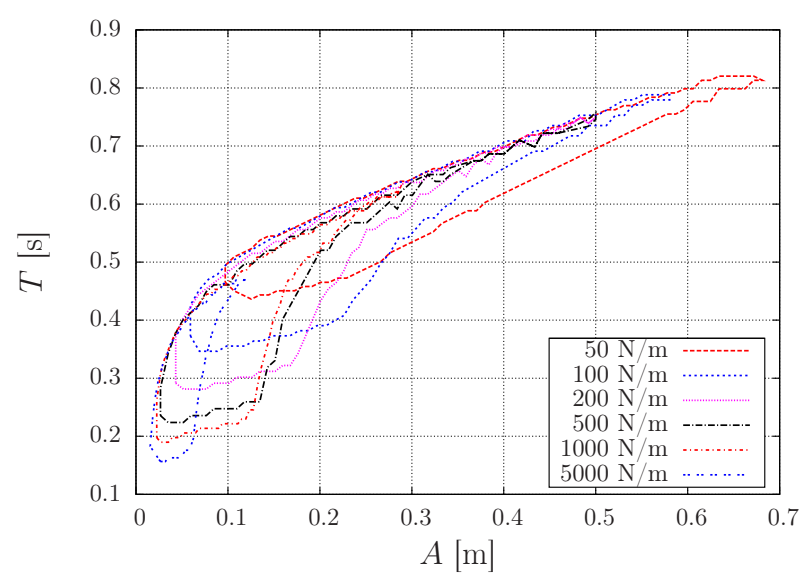

Fig. 14. Areas with stable cycles for different stiffnesses.

As the areas from Fig. 14 are not uniquely comparable w.r.t. their size and position, we inscribe a circle in each region, see Fig. 15. These circles can be interpreted as a robustness area when considering real robotic systems, which can suffer non-negligible tracking and sensor errors.

Table I summarizes the aforementioned results for the given example. It lists the position $\left(A_{C}\right.$ and $\left.T_{C}\right)$ and radius $r_{c}$ of the circle, the maximal robot velocity $\dot{x}_{C_{\max }}$ and acceleration $\ddot{x}_{C_{\max }}$ (resulting from the center of the circle), and the maximal power $P_{\max }$ and force $F_{\max }$ for the cycles from Fig. 10(a) in case of different stiffnesses. Clearly, the second large benefit of intrinsically elastic robots becomes clear: The significant reduction of contact forces, which leads to a load reduction for the physical robot.

Until now, the trajectory height $z_{0}$ remained the same. We examined mainly the case of dribbling at a given height. Another interesting aspect is the dribbling at a desired ball energy level that is related to the ball height and velocity. Figure 16(a) shows two ball trajectories for a cycle that starts on the same energy level but with different stiffnesses. Clearly, we need a significantly faster hand trajectory for the high stiffness case compared to an elastic (compliant) robot. 
TABLE I

COMPARISON

\begin{tabular}{c|c|c|c|c|c|c|c|c}
$K_{H}[\mathrm{~N} / \mathrm{m}]$ & $A_{C}[\mathrm{~m}]$ & $T_{C}[\mathrm{~s}]$ & $f_{C}[\mathrm{~Hz}]$ & $r_{C}$ & $\dot{x}_{C_{\max }}[\mathrm{m} / \mathrm{s}]$ & $\ddot{x}_{C_{\max }}\left[\mathrm{m} / \mathrm{s}^{2}\right]$ & $P_{\max }[\mathrm{W}]$ & $F_{\max }[\mathrm{N}]$ \\
\hline 50 & 0.2027 & 0.5200 & 2.4040 & 0.0538 & 1.5305 & 11.5590 & 13.6918 & 12.7022 \\
100 & 0.1715 & 0.4606 & 2.7137 & 0.0756 & 1.4617 & 12.4620 & 10.4531 & 11.4802 \\
200 & 0.1348 & 0.4045 & 3.0905 & 0.0767 & 1.3086 & 12.70533 & 10.5489 & 12.8450 \\
500 & 0.1474 & 0.4102 & 3.0470 & 0.0805 & 1.4113 & 13.5093 & 12.9995 & 17.0401 \\
1000 & 0.1045 & 0.3574 & 3.4972 & 0.0650 & 1.1485 & 12.6185 & 16.5415 & 22.2808 \\
5000 & 0.0583 & 0.3200 & 3.9066 & 0.0296 & 0.7153 & 8.7794 & 32.9895 & 44.7021
\end{tabular}

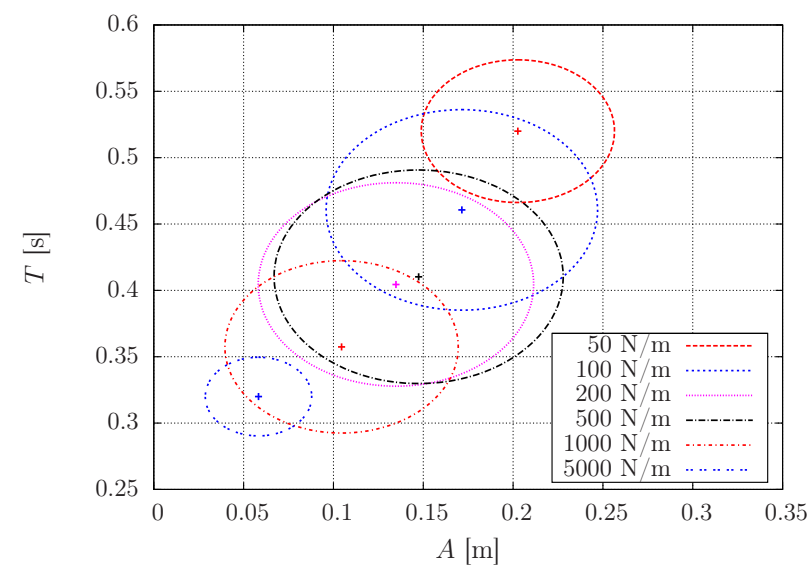

Fig. 15. Inscribed circles of the areas with stable cycles for different stiffnesses.
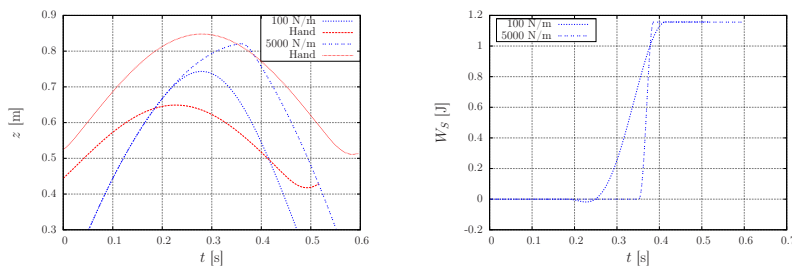

(a) Position of a cycle for the same (b) Energy evolution for a cycle at energy level with different stiff- the same energy with different stiffnesses. nesses.

Fig. 16. Dribbling at the same energy level with two different hand stiffnesses. Figure 16(a) shows the position of hand and ball. Figure 16(b) depicts the energy trajectory for both systems.

\section{CONCLUSION}

In this paper we developed a theoretical foundation for dribbling with an intrinsically elastic robot. We show the stability of a limit cycle with a perturbation approach and the cycle stability for a finite-time convergence sliding mode observer. This scheme is suitable to observe the ball motion even for the partially observable cycle with force sensing only, i.e. no vision information is necessary for our approach, though it could be easily integrated. Finally, we derived significant beneficial effects of intrinsic elasticity in terms of required peak power, impact force, and robustness compared to stiff actuation.

A video showing the application of the developed methods using a full $7 \mathrm{DoF}$ robot equipped with an intrinsically elastic hand is accessible from www.saferobots.com/dribbling.html. The according theory is given in [16]. Future work will extend the approach to two handed dribbling.

\section{ACKNOWLEDGMENTS}

This work has been partially funded by the European Commission's Sixth Framework Programme as part of the project VIACTORS under grant no. 231554 and SAPHARI under grant no. 287513. Furthermore, we would like to thank Mirko Kunze for his valuable contribution.

\section{REFERENCES}

[1] S. Haddadin, M. Weis, S. Wolf, and A. Albu-Schäffer, "Optimal control for maximizing link velocity of robotic variable stiffness joints," in accepted to: IFAC World Congress, 2011.

[2] M. Stilman. (without date) Mike Stilman: Previous Projects. Visited on 28th of february 2011. [Online]. Available: http://www.cc.gatech.edu/ mstilman/earlier.html

[3] D. Shiokata, A. Namiki, and M. Ishikawa, "Robot dribbling using a high-speed multifingered hand and a high-speed multifingered hand and a high-speed vision system," in 2005 IEEE/RSJ International Conference on Intelligent Robots and Systems, Edmont, Canada, 2005, pp. $3945-3950$.

[4] G. Bätz, M. Sobotka, D. Wolherr, and M. Buss, "Robot basketball: Ball dribbling - a modified juggling task," in 2008 IEEE International Conference on Robotics and Automation, San Diego, USA, 2009, pp. $2410-2415$

[5] U. Mettin, A. S. Shiriaev, G. Bätz, and D. Wolherr, "Ball dribbling with an underactuated continuous-time control phase," in 2010 IEEE International Conference on Robotics and Automation, Anchorage, USA, 2010, pp. $4669-4674$.

[6] M. Bühler, D. E. Koditschek, and P. Kindlmann, "A one degree of freedom juggler in a two degree of freedom environment," in Proceedings of the International Workshop on Intelligent Robots, Tokyo, Japan, 1988, pp. 91 - 97.

[7] R. Ronsse, P. Lefevre, and R. Sepulchre, "Rhythmic feedback control of a blind planar juggler," IEEE Transactions on Robotics, vol. 23, no. 4, pp. 790-802, 2007.

[8] P. Reist and R. D'Andrea, "Bouncing an unconstrained ball in three dimensions with a blind juggling robot," in 2009 IEEE International Conference on Robotics and Automation, Kobe, Japan, May 2009, pp. $1774-1781$.

[9] J. Yamaguchi, D. Nishino, and A. Takanishi, "Realization of dynamic biped walking varying joint stiffness using antagonistic driven joints," in IEEE Int. Conf. on Robotics and Automation (ICRA1998), Leuven, Belgium, 1998, pp. 2022-2029.

[10] M. Okada, S. Ban, and Y. Nakamura, "Skill of compliance with controlled charging/discharging of kinetic energy," in IEEE Int. Conf. on Robotics and Automation (ICRA2002), Washington, USA, 2002, pp. $2455-2460$.

[11] J. J. Fontanella, The Physics of Basketball. Baltimore, USA: John Hopkins University Press, 2006.

[12] R. C. Hibbeler, Engineering Mechanics. New Jersey, USA: Prentice Hall, 2010.

[13] (2010, October) Official basketball rules 2010 : Basketball equipment. Fédération Internationale de Basketball (FIBA). Visited on 14th of november 2010. [Online]. Available: http://www.fiba.com/downloads/Rules/2010/BasketballEquipment2010_V2.pdf

[14] S. V. Drakunov, "Sliding-mode observer based on equivalent control method," in Proceedings of the 31st Contefence on Decision and Control, Tuscon, USA, December 1992, pp. 2368 - 2369.

[15] K. H. Khalil, Nonlinear Systems. New Jersey, USA: Prentice Hall, 2002.

[16] S. Haddadin, K. Krieger, and A. Albu-Schäffer, "Exploiting elastic energy storage for cyclic manipulation: An analysis for basketball dribbling with an anthropomorphic robot," in accepted to: IEE/RSJ International Conference on Intelligent Robots and Systems, San Francisco, USA, 2011. 